\title{
Optimum ultrasound exposure condition for excitation of nonlinear behavior of microbubbles coated with surfactant
}

\author{
Shinichi Takeuchi, Kawan Soetanto, Toshio Sato and Norimichi Kawashima \\ Department of Biomedical Engineering, Toin University of Yokohama, Center of Advanced Research for Biomedical \\ Engineering (BME Center), Toin Human Science and Technology Center (Hustec), \\ Kurogane-cho 1614, Aoba-ku, Yokohama, 225-8502 Japan \\ e-mail:shin1@cc.toin.ac.jp
}

( Received 26 February 2001, Accepted for publication 17 May 2001 )

Keywords: Ultrasound exposure condition, Nonlinear behavior, Microbubbles, Surfactant, Harmonic imaging PACS number: 43.25.Ts, 43.25.Yw

\section{Introduction}

Ultrasound contrast agent for microbubbles coated with surfactant has been studied in our laboratory. The harmonic imaging method like ultrasound vascular image enhancement was proposed. This method allows to display clearly images of blood flow, blood vessel, cancer etc in which microbubbles are present [1,2]. A study on the nonlinear behavior of microbubbles and the development of double peak type ultrasound transducer (DPUT) with double peak type frequency characteristics for harmonic imaging system were reported [3]. Results on the ultrasound exposure method for harmonic imaging for microbubbles coated with surfactant are reported in this paper.

\section{Surfactant coated microbubbles}

Surfactant coated microbubbles of a size (diameter) distribution shown in Fig. 1 are used in this study. The size was distributed in the range of $0.7[\mu \mathrm{m}]-6[\mu \mathrm{m}]$ and the average size was $1.63[\mu \mathrm{m}]$. The properties of coated film are expressed in the shell elasticity parameter $S_{\mathrm{p}}$ and shell friction parameter $S_{\mathrm{f}}$. The frequency characteristics of ultrasound attenuation constant is expressed as the function of $S_{\mathrm{p}}$ and $S_{\mathrm{f}}$ [4] for transmission of bubble screen. The shell parameters $S_{\mathrm{p}}$ and $S_{\mathrm{f}}$ might be estimated by curve fitting method [4] to minimize the error between the calculated frequency characteristics of attenuation constant and experimental data.

The sample container filled with diluted microbubble liquid was inserted between the transmitting and receiving ultrasound probes. Then, the frequency characteristics of attenuation were measured by the transmission method. As a result, the values of $S_{\mathrm{p}}$ and $S_{\mathrm{f}}$ were estimated as follow.

$$
S_{\mathrm{p}}=0.3[\mathrm{~N} / \mathrm{m}], \quad S_{\mathrm{f}}=0.07[\mu \mathrm{Ns} / \mathrm{m}]
$$

The calculated curve of frequency characteristics of attenuation with these estimated values was shown, together with the experimented curve in Fig. 2.

\section{Consideration of ultrasound exposure condition}

\subsection{Method of consideration}

The ultrasound pulse exposure condition to maximize the generation efficiency of the 2 nd harmonic components based on the nonlinear behavior of microbubbles will be considered using numerical calculation. The nonlinear differential equation given in Eq. (1) was used for numerical calculation taking into consideration the effect of the compressibility of liquid surrounding bubbles. The condition to maximize the 2nd harmonic component excitation efficiency (ratio of 2nd harmonic component level to fundamental component level) will be calculated with Eq. (1) considering the frequency and the number of cycles in bursts of ultrasound pulse as the ultrasound exposure condition in this paper. For consideration of the effect of microbubble size distribution shown in Fig. 1 and concentration, the total scattered waveform was calculated by summing up all responded waves from microbubbles with each size in time domain. Then, frequency spectrum was calculated with the Fourier transform of this scattered waveform and the nonlinear response of microbubble was examined. The positions of microbubbles are always changing through their random walk movement, floating, and extinction in water. Thus, the positions of microbubbles in water are determined by using random number.

$$
\begin{gathered}
\left(1-\frac{\dot{R}}{C}\right) R \ddot{R}+\frac{3}{2} \dot{R}^{2}\left(1-\frac{\dot{R}}{3 C}\right)=\left(1+\frac{\dot{R}}{C}\right) \frac{P}{\rho}+\frac{R}{\rho C} \dot{P} \\
P=P_{\mathrm{g} 0}\left(\frac{R_{0}}{R}\right)^{3 \Gamma}+P_{\mathrm{v}}-P_{0}-\frac{2 \sigma}{R} \\
-2 S_{\mathrm{p}}\left(\frac{1}{R_{0}}-\frac{1}{R}\right)-\delta_{t}\left(R, S_{\mathrm{f}}\right) \omega \rho R \ddot{R}-P_{\mathrm{a}} \\
P_{\mathrm{g} 0}=P_{0}+\frac{2 \sigma}{R_{0}}-P_{\mathrm{v}}
\end{gathered}
$$$$
\delta_{t}=\frac{4 \mu}{\omega \rho_{0} R^{2}}+\frac{\frac{\omega R_{0}}{C}}{1+\left(\frac{\omega R_{0}}{C}\right)^{2}}+\left(\frac{\omega_{0}}{\omega}\right)^{2} B+\frac{S_{\mathrm{f}}}{4 \pi R^{3} \rho \omega}
$$

$B=(3 \Gamma-1)$

$$
\times\left[\frac{X(\sinh X+\sin X)-2(\cos X-\cos X)}{X^{2}(\cosh X+\cos X)+(3 \Gamma-1) X(\sinh X+\sin X)}\right]
$$

$X=R_{0} \sqrt{\frac{2 \omega \rho_{\mathrm{gA}}\left(1+2 \sigma / P_{0} R_{0}\right) H_{\mathrm{g}}}{K_{\mathrm{g}}}}$

Notations used in Eq. (1) are given below:

$R$ : Instantaneous radius of bubble, $R_{0}$ : Equilibrium radius of 


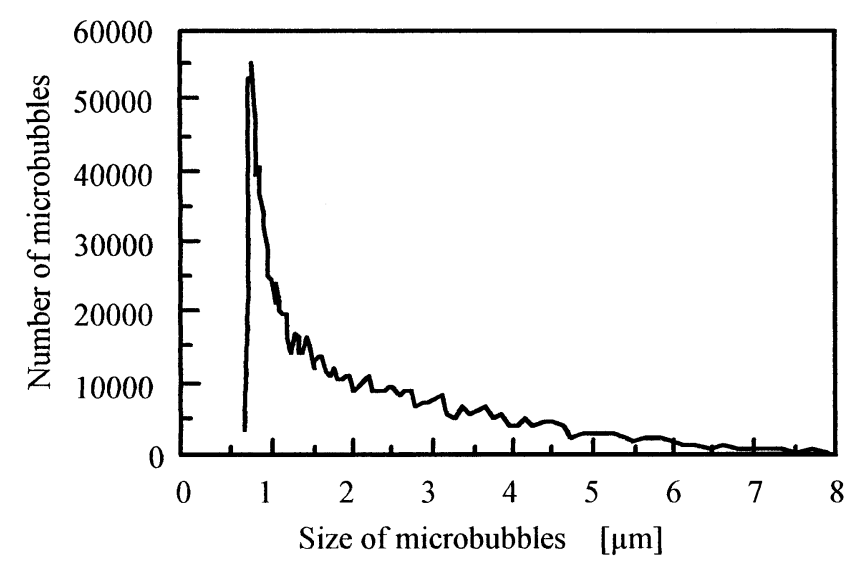

Fig. 1 Measured size (diameter) distribution data of surfactant coated microbubble used in this study.

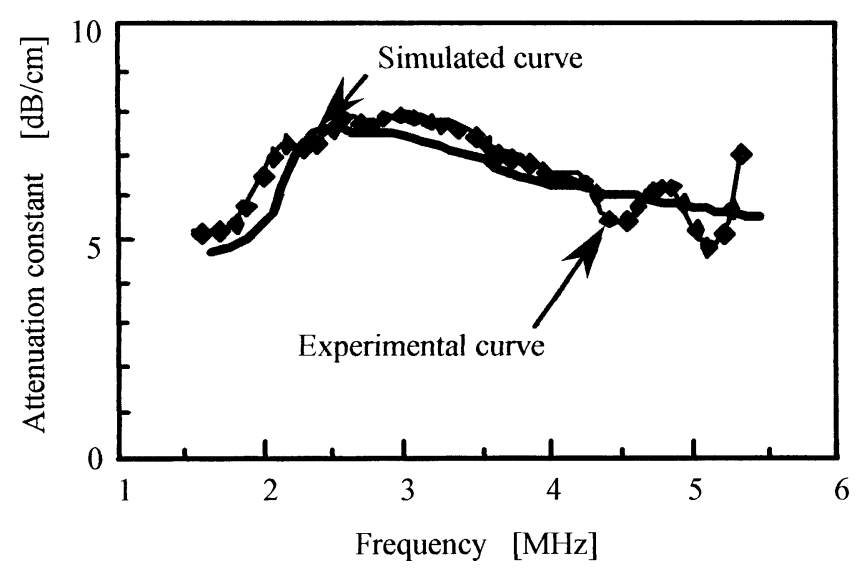

Fig. 2 Simulated and experimental frequency characteristics of attenuation constant of surfactant coated microbubbles.

bubble, $\rho$ : Density of surrounding liquid, $\rho_{\mathrm{gA}}$ : Free density of inner gas, $C$ : Sound speed in surrounding liquid, $P_{0}$ : Static pressure, $P_{\mathrm{v}}$ : Internal vaper pressure of bubble, $P_{\mathrm{a}}$ : Acoustic pressure of applied ultrasound, $\Gamma$ : Polytropic exponent of the gas, $\sigma$ : Surface tension of surrounding liquid, $\mu$ : Viscosity of surrounding liquid, $H_{\mathrm{g}}$ : Specific heat at constant pressure of gas, $K_{\mathrm{g}}$ : Thermal conductivity of inner gas, $S_{\mathrm{p}}$ : Shell elasticity parameter, $S_{\mathrm{f}}$ : Shell friction parameter.

3.2. Results

The relationship is shown in Fig. 3 between the illuminating frequency, the number of cycles in illuminating ultrasound burst to microbubbles and the 2nd harmonic component level included in scattered wave from microbubbles. The relationship between the illuminating frequencies, the number of cycles in bursts and the 2nd harmonic component excitation efficiency is shown in Fig. 4.

\section{Conclusion}

The peak of the 2nd harmonic level can be seen in the frequency range of $2 \mathrm{MHz}-2.5 \mathrm{MHz}$ in more than 4 cycles of illuminating ultrasound bursts, while the peak of the 2nd harmonic level can be seen near $1 \mathrm{MHz}$ in less than 4 cycles

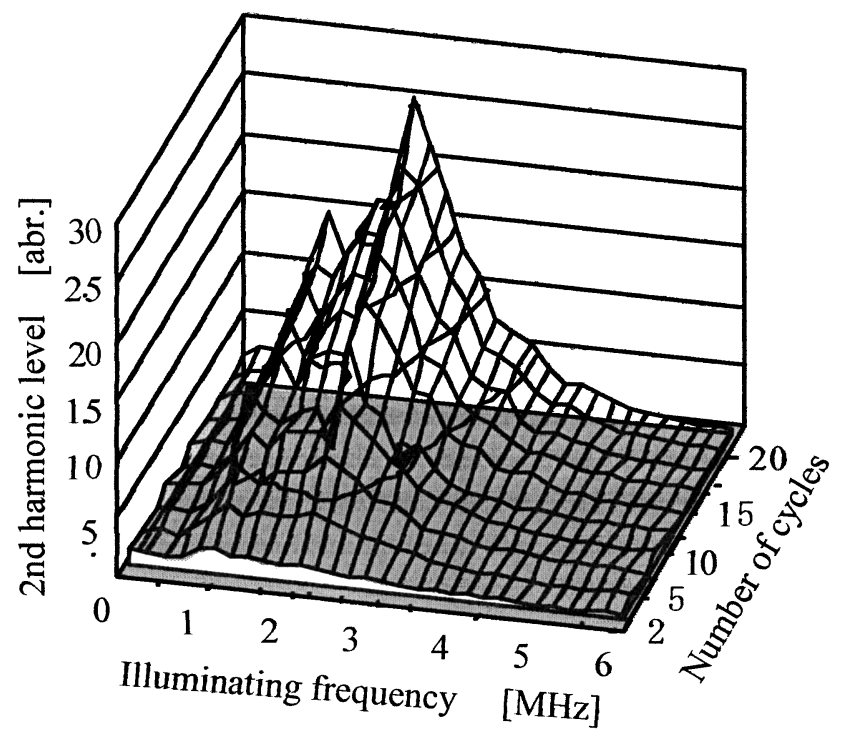

Fig. 3 Relationship between the illuminating frequency, the number of cycles in illuminating ultrasound bursts and 2nd harmonic component level included in scattered waves from microbubbles.

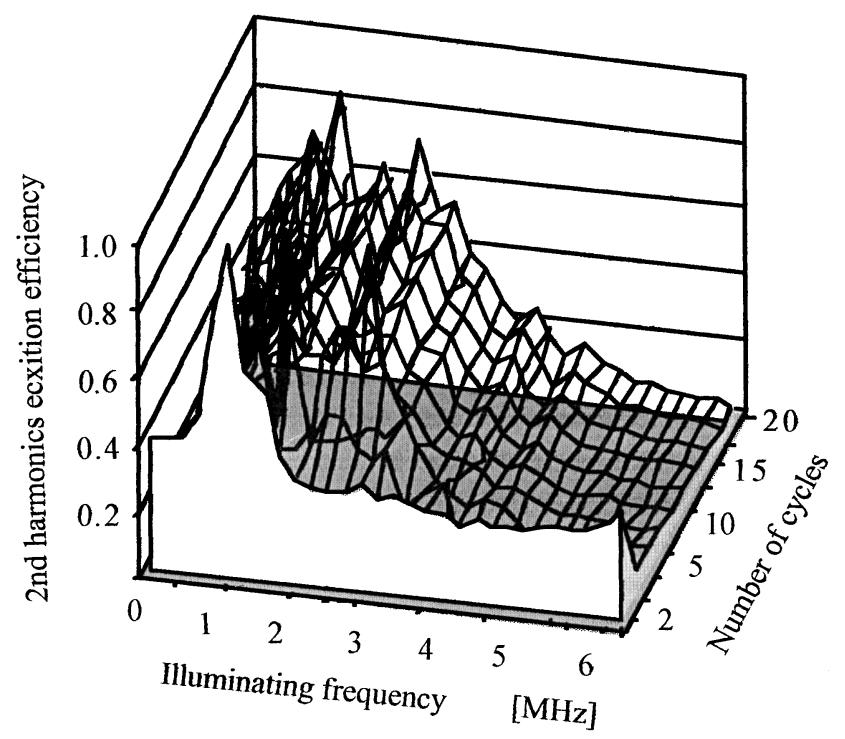

Fig. 4 Relationship between the illuminating frequency, the number of cycles in illuminating ultrasound bursts and 2nd harmonic component excitation efficiency included in scattered waves from microbubbles.

of bursts. The ultrasound attenuation coefficient by microbubbles shows a maximum value in the frequency range of $2 \mathrm{MHz}-2.5 \mathrm{MHz}$.

A similar level of the 2 nd harmonic component excitation efficiency is shown in either frequency. Furthermore, more cycles than above in ultrasound burst shows a higher 2nd harmonic component level.

It can be considered that ultrasound is scattered maximally by microbubbles in the frequency range of 2 MHz-2.5 MHz. The 2nd harmonic component frequency coincides with the maximum attenuation frequency at an 
ultrasound exposure frequency of 1 [MHz].

\section{References}

[1] S. Takeuchi, K. Soetanto and M. Okujima, "Numerical consideration of the relationship between nonlinear response and shell parameter of microbubble with coated membrane shell”, Jpn. J. Appl. Phys., 37, 3074 (1998).

[2] S. Takeuchi, K. Soetanto, K. Hayashi and M. Okujima, "The hybrid ultrasound transducer for ultrasound blood vascular image enhancement system", IECE Jpn., US97-11, 1 (1997).

[3] S. Takeuchi and K. Soetanto, "Detection of higher harmonic components scattered from microbubbles coated with surfactant membrane by double peak type ultrasound transducer", Proc. Autumn Meet. Acoust. Soc. Jpn., 993 (1999).

[4] S. Takeuchi and K. Soetanto, "Optimum condition of microbubble ultrasound contrast agent for harmonic imaging", 20th Anniv. Symp. Ultrasonic Electronics, 309 (1999). 